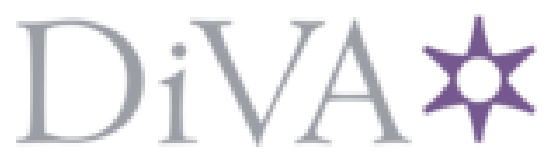

http://www.diva-portal.org

Postprint

This is the accepted version of a paper presented at 2012 IEEE International Symposium on Electrical Insulation (ISEI), 1O-13 June 2012, San Juan, PR, USA.

Citation for the original published paper:

Cheng, J., Werelius, P., Robalino, D., Ohlen, M. (2012)

Improvements of the Transformer Insulation XY Model Including Effect of Contamination

In: (pp. 169-174).

N.B. When citing this work, cite the original published paper.

Permanent link to this version:

http://urn.kb.se/resolve?urn=urn:nbn:se:kth:diva-303099 


\section{Improvements of the Transformer Insulation XY Model Including Effect of Contamination}

\author{
Jialu Cheng \\ Megger \\ Täby, Sweden \\ Jialu.cheng@megger.com \\ Diego Robalino \\ Megger \\ Dallas, USA \\ Diego.robalino@megger.com
}

\author{
Peter Werelius \\ Megger \\ Täby, Sweden \\ Peter.werelius@megger.com \\ Matz Ohlen \\ Megger \\ Täby, Sweden \\ Matz.ohlen@megger.com
}

\begin{abstract}
Dielectric Frequency Response, DFR (also known as Frequency Domain Spectroscopy, FDS), was introduced more than 20 years ago and has been thoroughly evaluated and proven in a number of research projects and field tests with good results. DFR data in combination with mathematical modeling of the oilpaper insulation is proven as an excellent tool for understanding insulation properties e.g. moisture content in cellulose insulation and temperature dependence of the insulation system. The model used to describe the insulation system inside transformers and bushings is called an XY-model. The model works well for most transformers but there are sometimes measurements showing an irregular response and not the expected shape for oil-paper insulation. In those cases, the moisture analysis becomes more difficult and sometimes almost impossible. One example is "frequency hump" phenomena sometimes observed in DFR measurements. A model of the internal creep currents from e.g. contamination has been developed and by applying it in moisture assessment software, better interpretation is obtained. Simulations are complemented with measurements on an actual $X Y$-cell with a conductive layer on the surface of the insulation. Results support the simulation model.
\end{abstract}

Keywords- cellulose; DFR; dielectric frequency response; FEM; insulation modeling; power transformer, contamination

\section{INTRODUCTION}

DFR (Dielectric Frequency Response) measurements on transformers and bushings in combination with insulation modeling are a good tool for investigating and understanding the properties of an insulating material. The model used to simulate the insulation system inside a transformer is usually called and described as an XY-model $[1,2]$. The insulation structure is represented by the relative amount of spacers (sticks) and barriers in the cooling duct as shown in Fig. 1. Parameter " $X$ " is defined as the ratio of the sum of all barriers in the duct, lumped together, and divided by the duct width. The spacer coverage " $Y$ ", is defined as the total width of all the spacers divided by the total length of the periphery of the duct.

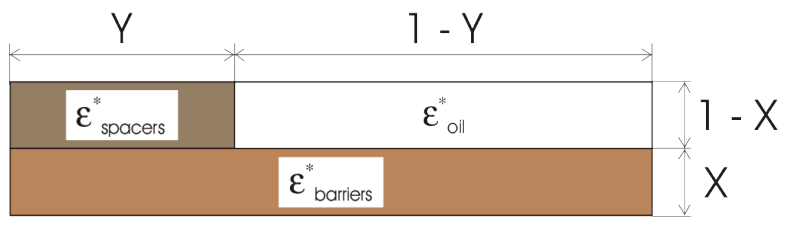

Figure 1. $\mathrm{XY}$ model as described in [1]

The permittivity of oil, $\varepsilon_{\text {oil }}$, spacers, $\varepsilon_{\text {spacers }}$ and barriers, $\varepsilon_{\text {barriers, }}$ are complex functions of both frequency and temperature.

\section{EQUIVALENT PERMITTIVITY CALCULATION OF THE XY MODEL}

\section{A. The prevailing capacitor model}

The total capacitance of the XY model as described in Fig. 2 can be calculated by four capacitors in series and parallel. The widely applied method is that the capacitors are in series in $\mathrm{X}$ direction and in parallel in $\mathrm{Y}$ direction, which is given by equation (1).

$$
\varepsilon(\omega, T)_{1}=\frac{Y}{\frac{1-X}{\varepsilon_{\text {spacers }}}+\frac{X}{\varepsilon_{\text {barriers }}}}+\frac{1-Y}{\frac{1-X}{\varepsilon_{\text {oil }}}+\frac{X}{\varepsilon_{\text {barriers }}}}
$$

A weakness of the above model is that it doesn't account for the electric field distortion, especially in the oil-cellulose interface near the "corner". Fig. 3 is a finite element method (FEM) simulation of the XY model consisting of pressboard and oil at $0.01 \mathrm{~Hz}$. The non-homogeneous distributed voltage potential lines indicate that the discontinuous effect may be cause for a significant error. 


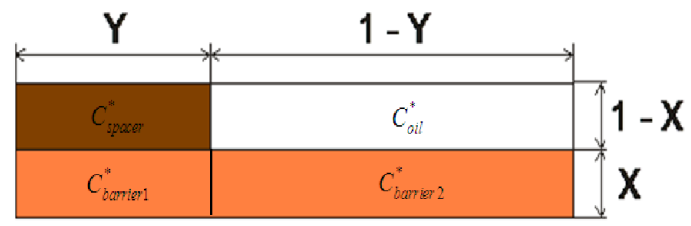

Figure 2. Capacitors representing the XY model



Figure 3. FEM Simulation of XY model for oil and pressboard at $0.01 \mathrm{~Hz}$

The relative error of the measured permittivity between the capacitor model (CM) and FEM is defined as:

$$
\text { Error }=(\text { FEM }-\mathrm{CM}) / \text { FEM }
$$

The real and imaginary permittivity errors as well as the dissipation factor error are plotted in in Fig. 4 for typical geometry and conductivity..

The relative error between the simple XY model and the FEM is typically up to $10 \%$.

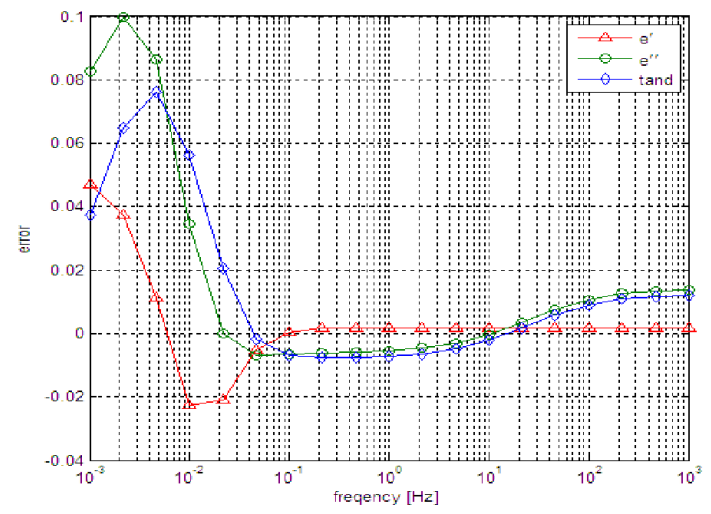

Figure 4. The relative errors with the typical insulation geometry $X=20 \%$ and $\mathrm{Y}=20 \%$, the oil conductivity sigma $=10^{-11} \mathrm{~S} / \mathrm{m}$ and the moisture content of the pressboard is $1 \%$.

\section{B. The modified capacitor model}

The widely applied capacitor model suggests that the capacitors are in series in $\mathrm{X}$ direction and parallel in $\mathrm{Y}$ direction. Due to the discontinuous effect, there also exists electric field which is in parallel with the winding electrodes. So the capacitors can be in parallel in $\mathrm{X}$ direction and in series in $\mathrm{Y}$ direction, which gives:

$$
\varepsilon(\omega, T)_{2}=\frac{1}{\frac{X}{Y \varepsilon_{\text {sticks }}+(1-Y) \varepsilon_{\text {barrier }}}+\frac{1-X}{Y \varepsilon_{\text {spacer }}+(1-Y) \varepsilon_{\text {oil }}}}
$$

Equations (1) and (3) are not equal. Given the same geometry and material, (3) results in an overestimation of permittivity whereas (1) gives an underestimation of permittivity compared to FEM simulations. An improved model equation is a combination of (1) and (3) which can be expressed by factor $\mathrm{K}$ :

$$
\varepsilon_{e q}=(1-K) \varepsilon_{1}+K \varepsilon_{2}
$$

By choosing a proper $\mathrm{K}$ factor, the relative error to FEM simulation is within 1\% which is shown in Fig. 5.. The factor $\mathrm{K}$ depends on geometry and oil conductivity. The improved model equation improves the accuracy especially at low frequencies where the oil duct field strength is low.

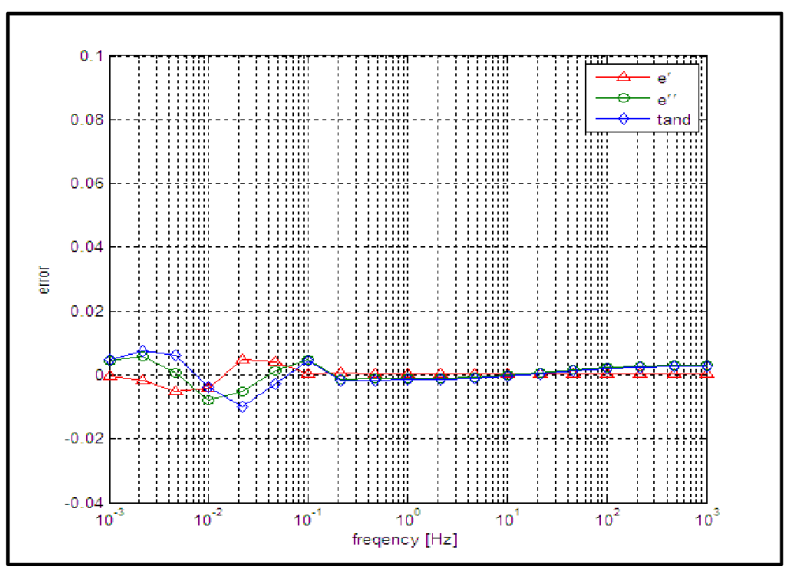

Figure 5. The relative errors of the modified capacitor model with $\mathrm{X}=20 \%$ and $\mathrm{Y}=20 \%$, the oil conductivity sigma $=10^{-11} \mathrm{~S} / \mathrm{m}$ and the moisture content of the pressboard is $1 \% . \mathrm{K}=0.3$ at frequencies below $0.1 \mathrm{~Hz}$ and $\mathrm{K}=0.12$ at frequencies above $0.1 \mathrm{~Hz}$.

\section{EFFECT OF CONTAMINATION ON DFR MEASUREMENT}

It is sometimes seen that DFR measurement on some transformers present atypical curves with a "hump" which can't be explained by the XY model. Fig. 6 is one example of the hump effect. The blue curve is the dielectric response of a transformer. The matching algorithm does not provide a good corresponding model and consequently the moisture estimation is not reliable. 


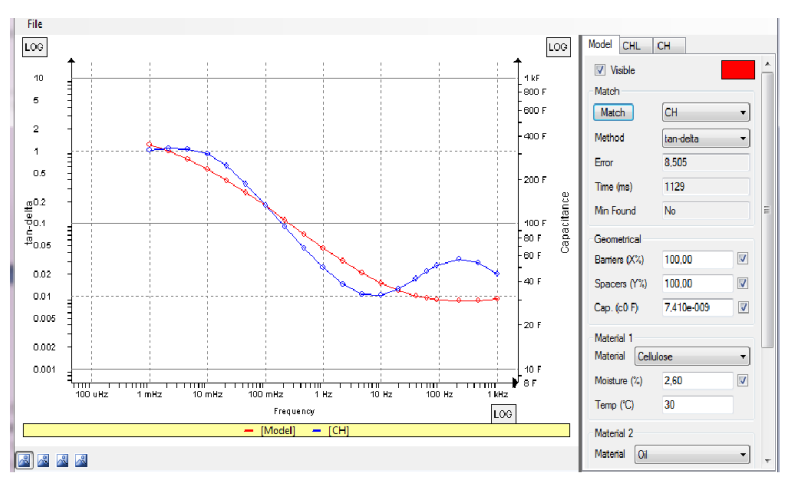

Figure 6. DFR measurements of power transformer. Lack of matching with model curve.

It is suggested by experienced DFR users that the hump is due to internal creep currents on the surface of the cellulose insulation caused by e.g. contamination.

\section{A. XY model used for FEM simulation}

The insulation structure of the power transformer is shown in Fig. 7. showing the pressboard barriers (horizontal), pressboard sticks/spaces (vertical) and the surrounding oil/air/vacuum (filled with color). Due to the symmetrical layout, a small fragment of air/oil and paper structure can be used in FEM calculations. The $\mathrm{X}$ and $\mathrm{Y}$ are specified for FEM calculations as shown in Fig. 8.

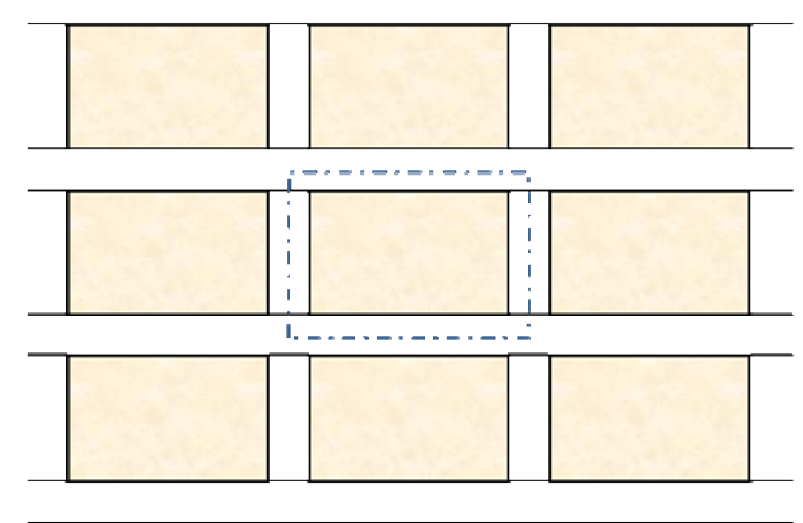

Figure 7. The insulation around the transformer winding

The contamination is assumed to be located at the interface of oil/air and pressboard. It is modeled as a thin layer with higher conductivity than oil. It could be distributed along the electrodes (in y direction) and/or across the electrodes (in $\mathrm{x}$ direction). The thickness, conductivity and permittivity (real number) of the contamination are varied to investigate the corresponding influence.

\section{B. Contaminations distribution in $x$ direction}

The $x$-direction distribution of the contamination layer is shown in Fig. 9. The simulation result with different thicknesses, conductivities and permittivities are shown in figures $10-12$. The simulation suggests that the contamination layer does add a 'hump' to the total response and the conductivity determines the hump's peak frequency.
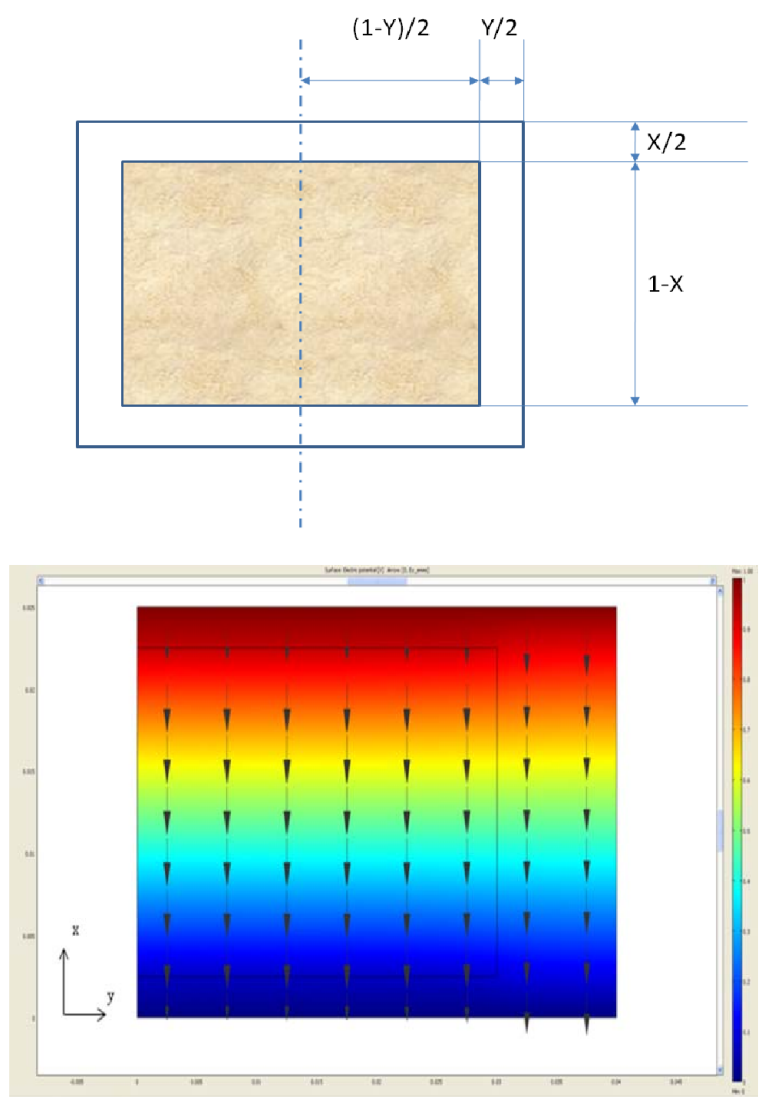

Figure 8. Modified XY model in FEM simulation software

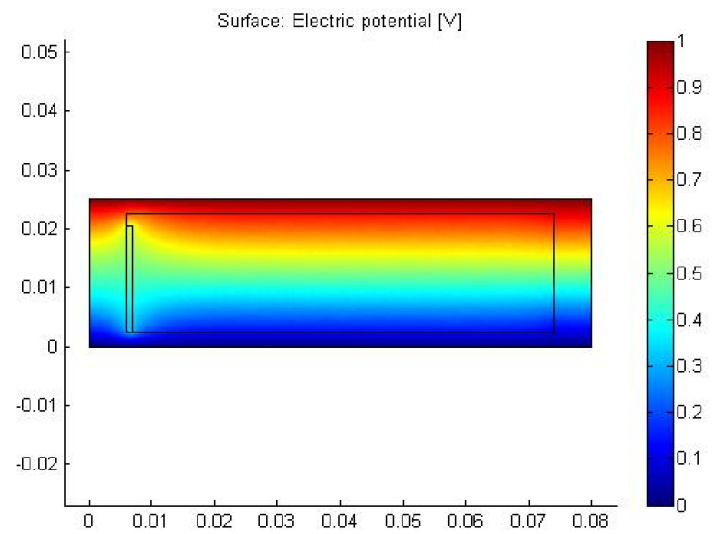

Figure 9. Distribution of the contamination layer perpendicular to the electrodes (x distribution)

Moreover, the increase of contamination thickness affects the peak frequency much more than the peak magnitude. With the variation of contaminant permittivity however, the total response remains the same. 




Figure 10. Dielectric response of the oil-paper system with contamination layers having different conductivities. Imaginary part of the relative complex permittivity. The thickness and permittivity of the contamination layer are kept constant

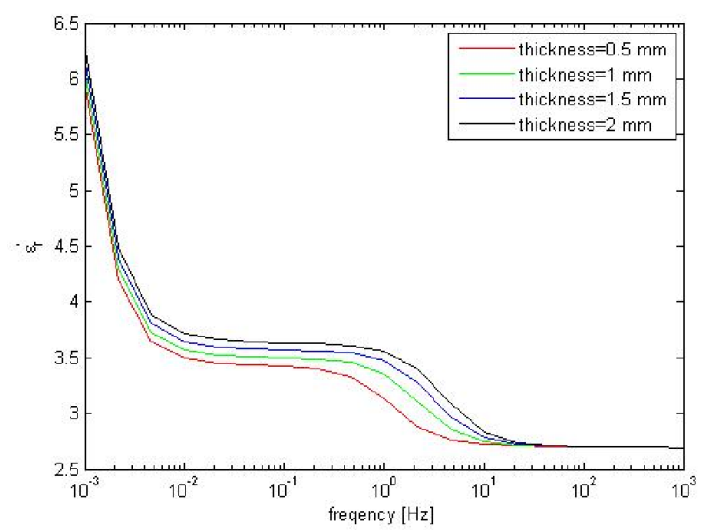

Figure 11. Dielectric response of the oil-paper system with contamination layers having different thicknesses. Imaginary part of the relative complex permittivity. The conductivity and permittivity of the contamination layer are kept constant

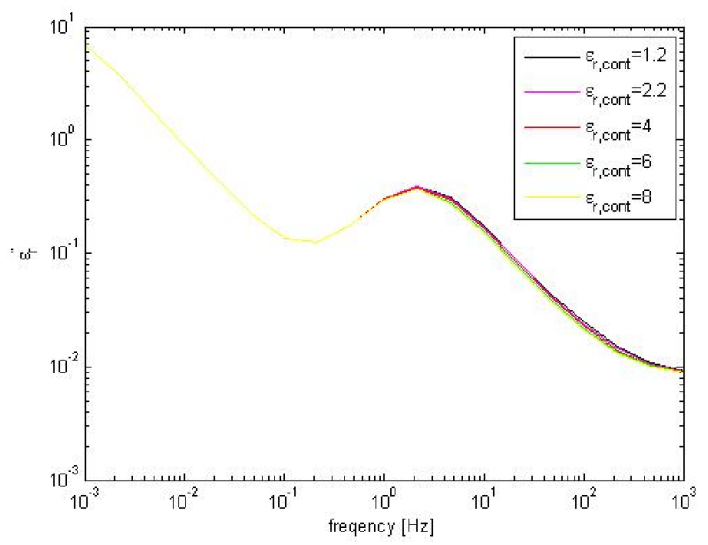

Figure 12. Spectrum of $\varepsilon$ " with different contaminant permittivity, The thickness and conductivity of the contamination layer are kept constant.

\section{Contamination distribution in y direction}

The y-direction distribution of the contamination layer is shown in Fig. 13. The simulation results with different thicknesses, conductivities and permittivities are shown in figures 14 - 16. Compared with $\mathrm{x}$-direction distribution, the thickness variation contributes to the peak magnitude instead of peak frequency. The permittivity variation contributes to both peak magnitude and peak frequency. The effect of the contamination distribution on the DFR measurement is summarized in Table I.

TABLE I. Comparison of contamination with different distribution

\begin{tabular}{|c|c|c|c|c|}
\hline \multirow{2}{*}{$\begin{array}{c}\text { Contamination } \\
\text { property }\end{array}$} & \multicolumn{2}{|c|}{ Peak magnitude } & \multicolumn{2}{c|}{ Peak frequency } \\
\cline { 2 - 5 } & $\mathrm{x}$ & $\mathrm{y}$ & $\mathrm{x}$ & $\mathrm{y}$ \\
\hline Conductivity & No & No & Yes & Yes \\
\hline Thickness & No & Yes & Yes & No \\
\hline Permittivity & No & Yes & No & Yes \\
\hline
\end{tabular}

The common features of two distributions are that with the increase of conductivity, the hump peak shifts to higher frequency. The permittivity and thickness of the contamination however have different features. The $\mathrm{x}$ direction contamination has higher loss due to more intense electric field which is perpendicular to the electrodes.

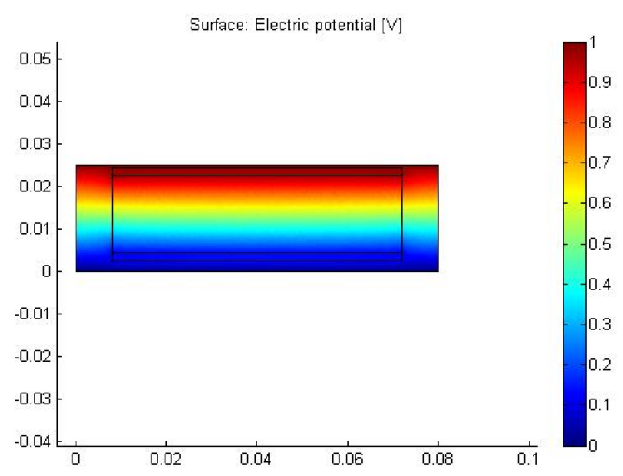

Figure 13. $\mathrm{y}$-Distribution of the contamination inside the $\mathrm{XY}$ model

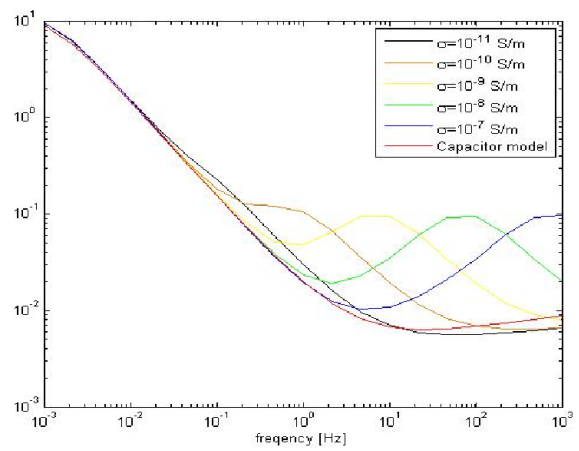

Figure 14. Dielectric response of the oil-paper system with contamination layers having different conductivities. Imaginary part of the relative complex permittivity. The thickness and permittivity of the contamination layer are 


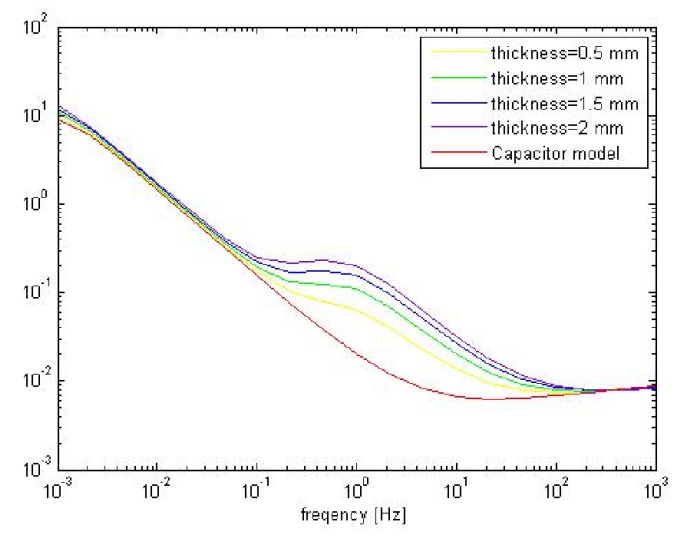

Figure 15. Dielectric response of the oil-paper system with contamination layers having different thicknesses. Imaginary part of the relative complex permittivity. The conductivity and permittivity of the contamination layer are kept constant.

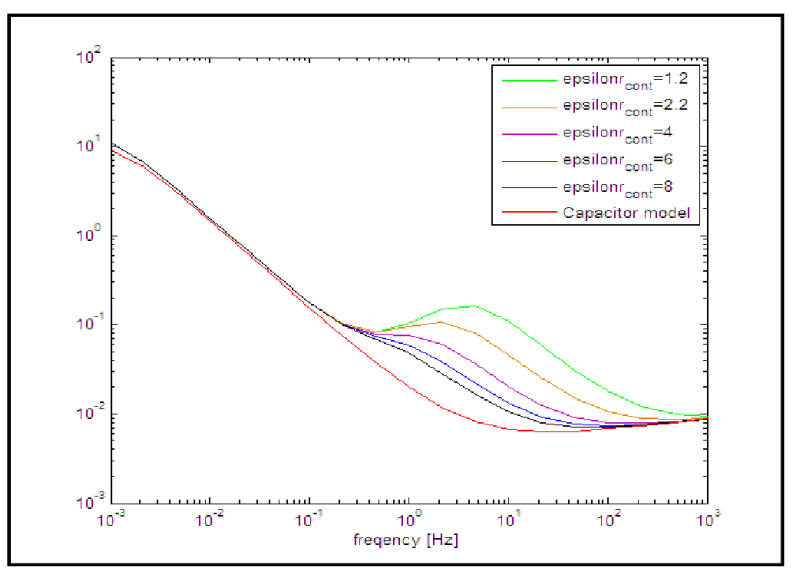

Figure 16. Spectrum of $\varepsilon^{\prime \prime}$ with different contaminant permittivity, the thickness of the contaminant layer is equal to $1 \mathrm{~mm}$, the contamination conductivity is equal to $3 \times 10^{-10} \mathrm{~S} / \mathrm{m}$. The thickness and conductivity of the contamination layer are kept constant.

\section{Contamination in stacked XY units}

The transformer insulation system consists of a lot of XY units. If a few units are contaminated, then the effect is averaged to each unit. Four units are simulated in FEM simulation software as shown in Fig. 17 and each unit has its own contamination. Fig. 18 is the result obtained from linearly varied conductive contamination, which has wider peak than single conductivity contamination. If there are two different kinds of contamination, the response is shown in Fig. 3.11, which has two separate peaks. Therefore, it is possible that the breadth of the hump is broader than pure Debye function and it is also possible that there are several humps.

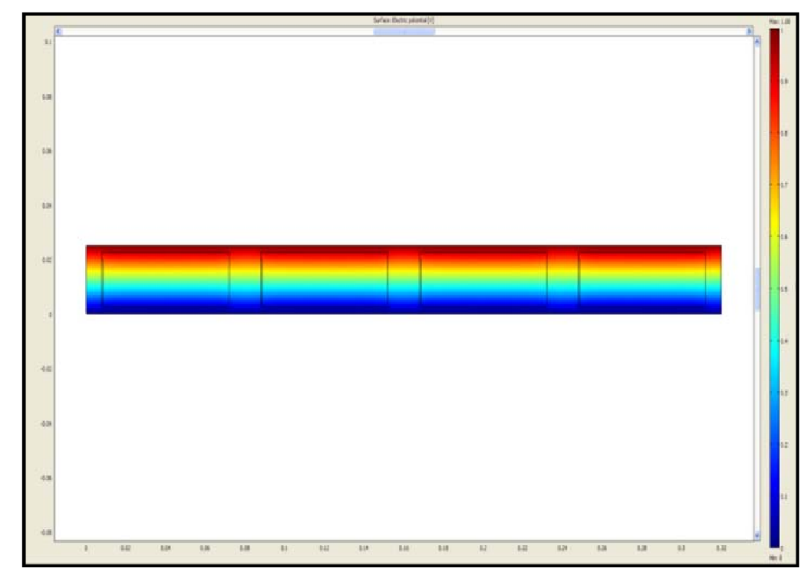

Figure 17. Hybrid distribution of the contamination layers



Figure 18. Dielectric response of the oil-paper system with contamination layers having linearly increased conductivities.

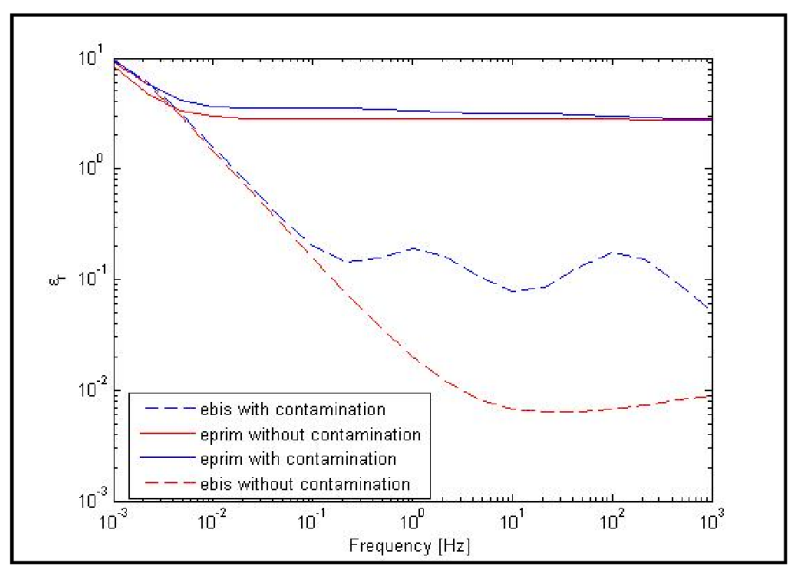

Figure 19. Dielectric response of the oil-paper system with contamination layers having two seperate conductivities. 


\section{EXPERIMENTAL RESULTS}

The contamination effect is experimentally demonstrated and presented herein. To observe the hump, the insulation material is required to be low loss. An insulation system shown in Fig. 20 is created by putting two PVC (Polyvinyl chloride) sheets together. The reasons for choosing PVC instead of pressboard are that PVC has the similar loss curve as dry pressboard and it is almost not influenced by ambient humidity.

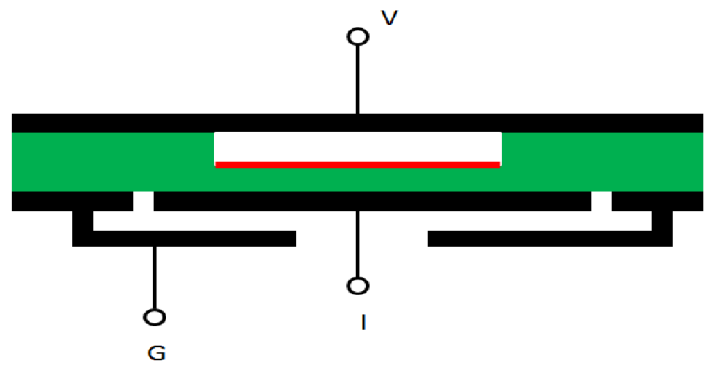

Figure 20. The constucted XY model consists of PVC and air.

A custom designed three-electrode test cell is used to measure the frequency response of the insulation. Furthermore, a contamination layer is added to the PVC sheet and the corresponding response is shown in Fig. 21. It is consistent with the simulation (Fig. 15) result. The interesting phenomenon is that real part permittivity with contamination is lower than that without hump, even if it has more polarization dipoles.



Figure 21. DFR from the PVC-air insulation system.

\section{CONTAMINATION MODELLING}

The simulation shows that the contamination behaves similar to a Debye function, which is given by equation (4). If multiple materials need to be modeled, an expansion function can be used (5).

$$
\varepsilon=\varepsilon_{\infty}+\frac{\Delta \varepsilon}{1+j \omega \tau}
$$

$$
\varepsilon=\varepsilon_{\infty}+\sum_{n=1}^{p} \frac{\Delta \varepsilon_{n}}{1+j \omega \tau_{n}}
$$

The Debye model can be implemented in the XY-model and implemented in the matching algorithms. Figure 22 shows the results from modeling the same transformer presented in Fig. 6. The DFR measurement is matched again including the contamination model. A good analysis can be obtained and the moisture content in this transformer is estimated to $1.7 \%$.

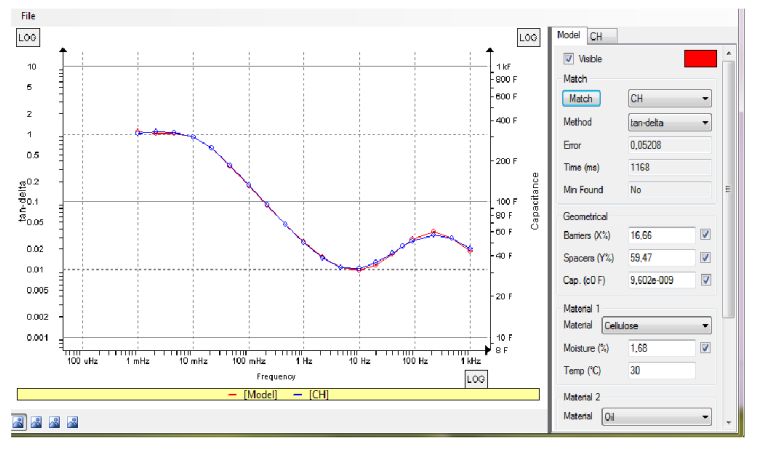

Figure 22. A new analysis including contamination parameters for the transformer with irreglular response

\section{CONCLUSIONS AND DISCUSSION}

The traditional XY model does not include effects of nonuniform electric field. Simulations using FEM indicate that errors may be significant at lower frequencies especially in the case that the permittivity difference between oil and cellulose is large. The improved model is able to give more accurate solution by including a compensation factor $\mathrm{K}$. The factor is rather complex and a function of geometry, oil conductivity and frequency. Further work is necessary to investigate to what extent this compensation may improve the accuracy of the moisture assessment process.

Surface currents due to e.g. contamination can sometimes occur and may create a "hump" in the frequency response. The FEM simulation reveals the basic properties of the effect of a conductive layer on the surface of cellulose. The response can be described by Debye functions with relaxation frequency pending conductivity, permittivity and thickness of the conductive layer.

\section{REFERENCES}

[1] U. Gäfvert, L. Adeen, M. Tapper, P. Ghasemi, B. Jönsson, "Dielectric Spectroscopy in Time and Frequency Domain Applied to Diagnostics of Power Transformers", Proc. of the $6^{\text {th }}$ ICPADM, Xi' an, China, 2000.

[2] U. Gäfvert, G. Frimpong and J. Fuhr, "Modeling of Dielecric Measurements on Power Transformers", Paper 15-103, CIGRE Session Paris 